\title{
Sewer Networks Optimization Using Cellular Automata
}

\author{
Maryam Rohani ${ }^{1}$, Mohammad Hadi Afshar ${ }^{1}$ \\ ${ }^{1}$ School of Civil Engineering, Iran Univ. of Science and Tech., Tehran, Iran \\ Correspondence: Maryam Rohani, PhD Student, School of Civil Engineering, Iran Univ. of Science and Tech., Tehran, \\ Iran. Tel: 98-9123162480. E-mail: mrohani@iust.ac.ir
}

Received: October 15, 2013 Accepted: October 27, 2013 Available online: November 7, 2013

doi:10.11114/set.v1i1.237

URL: http://dx.doi.org/10.11114/set.v1i1.237

\begin{abstract}
The Hybrid Cellular Automata (HCA) method is used in this paper for the optimal design of sewer network problems with the fixed layout. The HCA method decomposes the problem into two sub-problems with considering the pipe diameters and nodal cover depths as decision variables. Two stages are solved iteratively for determining the decision variables in a manner to minimize the total cost of the sewer network subject to the operational constraints. The HCA method is used to optimally solve three benchmark examples with different sizes and the results are presented and compared to those of the existing methods. The results indicate that the HCA method is more efficient and effective than the alternative methods.
\end{abstract}

Keywords: Sewer Network, Optimization methods, Cellular Automata, design problem.

\section{Introduction}

Sewer network systems as a necessary urban infrastructure play an important role in the urban areas. The main objective of optimal sewer network design problem is minimization of the capital investment on infrastructure whereas ensuring good performance under specified design criteria. This topic has received considerable attention and different numerical optimization approaches have been introduced and applied to the optimal design of sewer networks (Afshar, Shahidi, Rohani, \& Sargolzaei, 2011, Afshar \& Rohani, 2012). These include the Linear Programming (LP), Non-Linear Programming (NLP), Dynamic Programming (DP), and Evolutionary Algorithms.

There have been some attempts using the Linear Programming method to solve the problem of sewer network design, such as Deininger (1970), Dajani and Gemmell (1971), Froise and Burges (1978), and Walters and Templeman (1979). Gupta, Agarwal, \& Khanna (1976), Lemieux, Zech, \& Delarue (1976), and Price (1978) applied NLP and Swamee (2001) used the Lagrange multiplier method to yield optimal sewer network design.

Among these methods, DP is the mostly used method for the optimal design of the sewer networks due to serial features of these networks. Merrit and Bogan (1973), Mays and Wenzel (1976), Robinson and Labadie (1981), Yen, Cheng, Jun, Voohees, \& Wenzel (1984), and Kulkarni and Khanna (1985) applied DP for the optimal design of wastewater and/or storm water networks. Although, DP methods are theoretically capable of finding the global optimum solution, but they suffer from the curse of dimensionality limitation and therefore are not appropriate method for the large scale real-world sewer networks.

Recently, Evolutionary approaches are being used for the problem due to their simplicity and flexibility for both continuous and discrete problems without any assumption about the optimization objectives and good results have been reported using these methods. Heaney, Wright, Sample, Field, \& Fan (1999) had used Genetic Algorithm (GA) on spreadsheet templates to get near-optimal solutions for the optimal design of sewer networks. Liang, Thompson, \& Young (2004) applied GA and Tabu Search (TS) algorithm for the optimal design of sewer networks. Afshar (2007) proposed the sequential feature of solution construction in the Ant Colony Optimization Algorithm (ACOA) to develop two partially constrained ACO algorithms for the solution of storm sewer network design problems. Pan and Kao (2009) had integrated Quadratic Programming (QP) with GA to solve the sewer network optimization problem. The applicability and efficiency of the GA-QP model were tested and the results indicated that the GA-QP model could obtain various near optimum design alternatives within an acceptable computational time. Wang and Zhou (2009) analyzed and compared the performances of GA, Particle Swarm Optimization (PSO) and Ant Colony Algorithms (ACA) from three aspects of convergence, speed and complexity. It was shown that ACA is superior to other methods 
for better convergence and satisfactory speed. Afshar (2010) applied the Continuous Ant Colony Optimization Algorithm (CACOA) for the optimal design of sewer networks. Yeh, Chu, Chang, \& Lin (2011) applied TS and Simulated Annealing (SA) for the optimization of sewer network problems.

Since the basic theories of Evolutionary Algorithms, emulating the natural optimization process of evolution, are similar to the natural evolutionary process, they need a big search space in spite of intelligent process, and so they involve high computational costs and need a large number of iteration and computational efforts to achieve the optimum solutions. Moreover, they require some free parameters that should be sensitive analyzed for obtaining the optimal solution.

Cellular Automata (CA), a novel optimization algorithm, has recently introduced and attracted much attention and has been widely applied to some engineering problems.

In this paper, Hybrid CA (HCA) methods are used for the design of sewer network with the fixed layout. In the HCA methods, the problem is decomposed in two stages solved iteratively to get the results. In the first stage, nodal cover depths of the network are determined with the fixed values for pipe diameters using a CA method with the nodes considered as the CA cells and nodal cover depths as cell states. In the second stage, the obtained nodal cover depths in the first stage are used to calculate the pipe diameters with another CA method. In this stage, the pipes considered as the CA cells and their diameters as the cell states. Two different updating rules, Continuous and Discrete approaches, are used for CA updating rule of the second stage depending on the treatment of the pipe diameters. The CA updating rule is derived by requiring that the network cost is minimized in the neighborhood of each cell. The HCA methods are used to design three benchmark examples and comparison the results with the existing ones show the efficiency and effectiveness of the methods to solve the sewer design optimization problems.

\section{Cellular Automata}

Cellular Automata (CA), a model of self-reproducing system, was conceived by Ulam (1960) and Von Neumann (1966) and later completed and improved with the work of other researchers like Thatcher (1964), Codd (1968), and Burks (1972).

CA has a set of identical elements, called cells with finite possible value called cell state. The new states of all cells are defined simultaneously using an updating rule, which is a function of the previous state of the cell itself and its neighborhoods.

CA had been used as a simulator in various fields such as computer science, (Wolfram 1988), chemistry (Packard, 1986), and medical profession (Sentos \& Coutinho, 2001). Recently, some research showed that CA can be used as a practical and efficient optimization engine, which relies on the key properties of: locality of the neighborhoods interactions, homogeneity of the evolving mechanism, parallelism of the computation, and simplicity of the model structure. CA has been extensively used as a viable and efficient optimization algorithm for the structural design (Kita \& Toyoda, 2000, Missoum, Gürdal, \& Setoodeh, 2005, and Setoodeh, Gürdalb, \& Watson, 2006), estimating shortest path (Adamatzky 1996) or trip distribution problems (El Dessouki, Fathi, \& Rouphail, 2001), and computer networks (Shuai \& Zhao, 2004).

In the early applications in water resource problems, CA was used to produce good initial populations for a GA leading to improved performance of the GA (Keedwell \& khu, 2005, Guo, Keedwell, Walters, \& Khu, 2007a). The first use of CA as a stand-alone optimizer was demonstrated by Guo, Walters, Khu, \& Keedwell (2007b) for optimal design of storm sewer networks based on the simplifying assumption of known slopes. Afshar and Shahidi (2009) were the first to propose CA with mathematically derived transition rules for the optimal water supply and hydropower operation of a single reservoir. Later, Afshar et al. (2011) proposed a single stage CA for the optimal design of sewer networks with fixed layout using the nodal excavation depth as the decision variables of the problem. Afshar and Rohani (2012) extended the single stage CA method of Afshar et al. (2011) to two stage CA and proposed Hybrid CA, in which nodal cover depths and pipes diameter were considered as decision variables.

\section{Sewer Network Size Optimization}

Sewer network system is one of the urban infrastructure systems with huge construction and operation cost and any attempt to reduce these costs result in considerable saving. A sewer network is an underground system built to convey waste water to one or more collection points (outfalls).

Optimal sewer network design with a fixed layout aims to find a cost-effective solution by determining the pipes diameters and slopes which minimizes the capital investment whilst ensuring a good system performance under specific design criteria. The problem of sewer network design for a fixed network layout, in the absence of pumps and drops, can be formulated as:

$$
\text { Min } C_{\text {network }}=\sum C p_{l}+\sum C m_{i}=\sum L_{l} K p\left(D_{l}, X_{i}, X_{j}\right)+\sum K m\left(h m_{k}\right)
$$


Subject to:

$$
\begin{array}{lc}
V_{\min } \leq V_{l} \leq V_{\max } & l=1, \ldots, N L \\
\beta_{\min } \leq \beta_{l} \leq \beta_{\max } & l=1, \ldots, N L \\
S_{\min } \leq S_{l} \leq S_{\max } & l=1, \ldots, N L \\
X_{\min } \leq X_{k} \leq X_{\max } & k=1, \ldots, N N \\
D_{l} \in \boldsymbol{D} & l=1, \ldots, N L \\
D_{l} \leq D_{l}^{\prime} & l=1, \ldots, N L
\end{array}
$$

Where, $C_{\text {network }}$ is the total cost of the network, $C p_{l}$ is the installation cost of $l^{\text {th }}$ pipe, $C m_{i}$ is the cost of $i^{\text {th }}$ manhole, $N L$ is the number of pipes in the network, $N N$ is the number of nodes in the network, $L_{l}$ is the length of $l^{\text {th }}$ pipe, $K p$ is the unit cost of $l^{\text {th }}$ pipe defined as a function of its diameter $\left(D_{l}\right)$ and upstream and downstream nodal cover depth $\left(X_{i}, X_{j}\right)$, and $\mathrm{Km}$ is the cost of manhole construction as a function of manhole depth $(\mathrm{hm})$.

Equations (2) to (7) represents the constraints of velocity, water-depth ratio, pipe slope, nodal cover depth, commercially available pipe diameter, and progressive diameter for the sewer network problem, respectively, where, $V_{l}$ is the velocity of $l^{\text {th }}$ pipe, $\beta_{l}=y_{l} / D_{l}, y_{l}$ is the flow depth of $l^{\text {th }}$ pipe, $S_{l}$ is the slope of $l^{\text {th }}$ pipe, $X_{k}$ is the cover depth of $k^{\text {th }}$ node, $\boldsymbol{D}$ is the set of commercially available pipe diameters, $l$ refers to the set of pipe located downstream of pipe $l$, and min, $\max$ are the allowable minimum and maximum parameters, respectively.

\section{Hybrid Cellular Automata (HCA)}

Application of CA to any problem requires that four basic components of the CA method, cell, cell state, neighborhood, and updating rule, are properly defined. In this paper, pipe diameters and nodal cover depths are chosen as the decision variables.

The Hybrid Cellular Automata (HCA) formulation requires decomposing the problem into two sub-optimization problems which are solved iteratively in two stage manners. In the first stage, each node of the sewer network is regarded as a cell and nodal cover depths are considered as the cell states, which are determined with the fixed values of pipe diameters. In the second stage, the pipe diameters are calculated by solving a second nonlinear sub-optimization problem with considering the calculated nodal cover depths from the first stage as fixed values. Two different updating rules, continuous and discrete, are derived and used depending on the treatment of pipe diameters. In the continuous approach, the pipe diameters are considered as continuous variables and the corresponding updating rule is derived mathematically from the original objective function of the problem and followed by a rounding process in which the continuous pipe diameters calculated are rounded, if required, to find the discrete optimal pipe diameters, while in the discrete approach, an ad-hoc updating rule is derived based on the discrete nature of pipe diameters. The described two stage process is iterated until convergence is achieved.

Considering the nodal cover depths, $X_{k} ; k=1, \ldots, N N$, and pipe diameters, $D_{l} ; l=1, \ldots, N L$, as the decision variables, these constraints can be easily applied as box constraints. Using a penalty method for satisfaction of remaining constraints, the total penalized objective function of the sewer network optimization problem can be defined as follows:

$$
\text { Min } C=\sum C p_{l}+\sum C m_{i}+\sum\left(\alpha C S V v_{l}+\alpha C S V s_{l}+\alpha C S V \beta_{l}\right)
$$

Where, $\alpha$ is the penalty parameters with large enough positive value, and $\operatorname{CSV} v, \operatorname{CSV}$, CSV $\beta$ represent the violation from the constraints of velocity, slope, and water-depth ratio for each pipe, respectively, $C S V v_{l}=\left(1-V_{l} / V_{\min }\right)^{2}+\left(V_{l} / V_{\max }-1\right)^{2}$, $C S V \beta_{l}=\left(1-\beta_{l} / \beta_{\min }\right)^{2}+\left(\beta_{l} / \beta_{\max }-1\right)^{2}, C S V s_{l}=\left(1-s_{l} / s_{\min }\right)^{2}+\left(s_{l} / s_{\max }-1\right)^{2}$.

Subject to:

$$
\begin{array}{cc}
X_{\min } \leq X_{k} \leq X_{\max } & k=1, \ldots, N N \\
D_{l} \in \boldsymbol{D} & l=1, \ldots, N L
\end{array}
$$

The process is started with arbitrary sets of pipe diameters and nodal cover depths satisfying the constraints of (9) and (10).

\subsection{First Stage}

In the first stage, the nodal cover depths are calculated with the minimization of the following local objective function over the cell neighborhood $\left(\Omega_{k}\right)$ considering the fixed values for the pipe diameters:

$$
C_{k}=\sum\left(C p_{l}+\alpha C S V v_{l}+\alpha C S V s_{l}+\alpha C S V \beta_{l}\right)+C m_{k}
$$


Subject to the box constraints of $X_{\min } \leq X_{k} \leq X_{\max }$.

Minimization of the local objective function of Equation (11) with respect to the nodal cover depth $\left(X_{k}\right)$ leads to the nonlinear equations to be solved with the Newton-Raphson method which results in the updated nodal cover depths defined as:

$$
F\left(X_{k}\right)=\partial C_{k} / \partial X_{k}=0 \quad \Delta X_{k}=-F_{k} /\left.(\partial F / \partial X)_{k}\right|^{k k} \quad \Delta X_{k}=\Delta X_{k}^{k k+1}-\Delta X_{k}^{k k}
$$

Where, $F_{k}^{k k}=F\left(X_{k}^{k k}\right), k k$ is the nonlinear iteration index, and $\Delta X_{k}$ is the change in the value of the cell state. $F_{k}$ and $(\partial F / \partial X)_{k}$ are both implicit functions of the $X_{k}$ which can be calculated using the chain rule of differentiation and Manning equation. This procedure is repeated for the cell under consideration until the convergence is met and the process of updating is repeated for all cells of the network at the end of which the first stage is terminated.

\subsection{Second Stage}

In the second stage, the values of nodal cover depths obtained in the first stage are used to get the optimal pipe diameters. Two CA approaches are applied to solve this problem, in which the pipes and corresponding diameters are considered as the CA cell and cell state, respectively. The neighborhood of the CA is simply considered as the cell itself without any neighboring cells.

\subsubsection{Discrete Approach}

In discrete method, the pipe diameters are treated as discrete values leading to an ad-hoc CA updating rule derived based on engineering judgment. The diameter of each pipe is changed such that the pipe cost is minimized in a manner to satisfy the constraints of velocity and water depth ratio. The following three engineering based ad-hoc updating rule is used to update the cell state:

1) If constraints of velocity and water-depth ratio are all satisfied, pipe diameter is decreased to the lower diameter available to minimize the objective function.

2) If one or both of the maximum velocity and water-depth ratio constraints are violated, the pipe diameter is increased to the upper diameter available.

3) If one or both of the minimum velocity and water-depth ratio constraints are violated, the pipe diameter is decreased to the lower diameter available.

\subsubsection{Continuous Approach}

In continuous method, the updating rule is derived mathematically assuming pipe diameter as continuous variable followed by a rounding procedure to convert the continuous solutions to discrete available diameters such that the following localized objective function is minimized:

$$
\text { Minimize } C_{l}=\mathrm{Cp}_{l}+\alpha C S V v_{l}+\alpha C S V \beta_{l}+\mathrm{Cm}_{i}+\mathrm{Cm}_{j}
$$

Subject to the box constraints of:

$$
D_{\min } \leq D_{l} \leq D_{\max }
$$

Where $i$ and $j$ refer to the upstream and downstream node of $l^{\text {th }}$ pipe, $D_{\min }$ and $D_{\max }$ represent the minimum and maximum components of available commercial diameters, respectively. The updating rule is derived mathematically by requiring that the objective function of Equation (13) is stationary with respect to the cell state with applying the Newton-Raphson linearization:

$$
G_{l}=\partial C_{l} / \partial D_{l}=0 \quad \Delta D_{l}=-G_{l} /(\partial G / \partial D)_{l} \quad \Delta D_{l}=\Delta D_{l}^{k k+1}-\Delta D_{l}^{k k}
$$

This procedure is repeated for the cell under consideration until the convergence is met. Once the process of updating of all cell states are completed, the continuous diameters so calculated are rounded to the upper discrete diameters available before returning to the first stage. More details of the method can be found in the work of Afshar and Rohani (2012). 


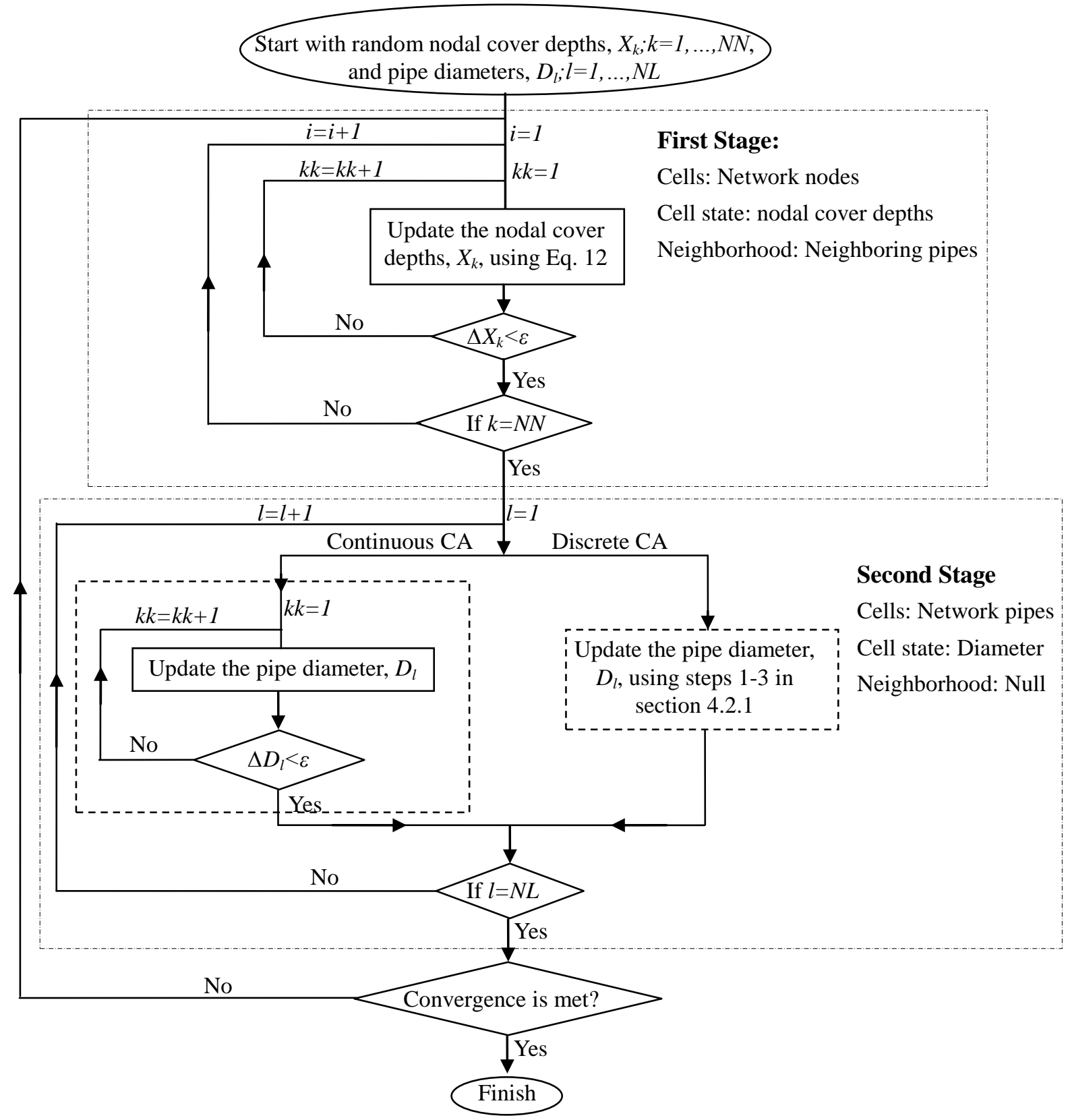

Figure 1. Schematic description of the HCA method.

A schematic description of the HCA method is illustrated in Figure 1 for both discrete and continuous versions in which the cells, cell states, neighborhood and the updating rules are briefly described for more clarification.

\section{Test Examples}

The performance of the HCA method is investigated in this section by applying the model to three design problems with different sizes in the literature. These hypothetical test examples were previously proposed and used by Moeini and Afshar (2012 a,b) for the simultaneous layout and size optimization of sewer network using ACOA based methods. 


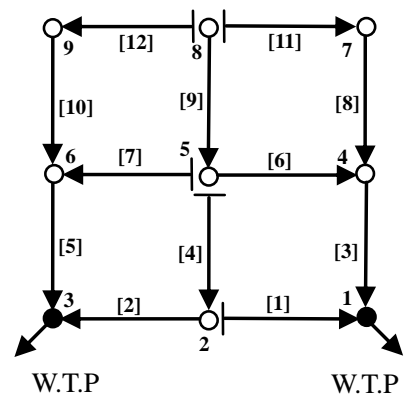

(a)

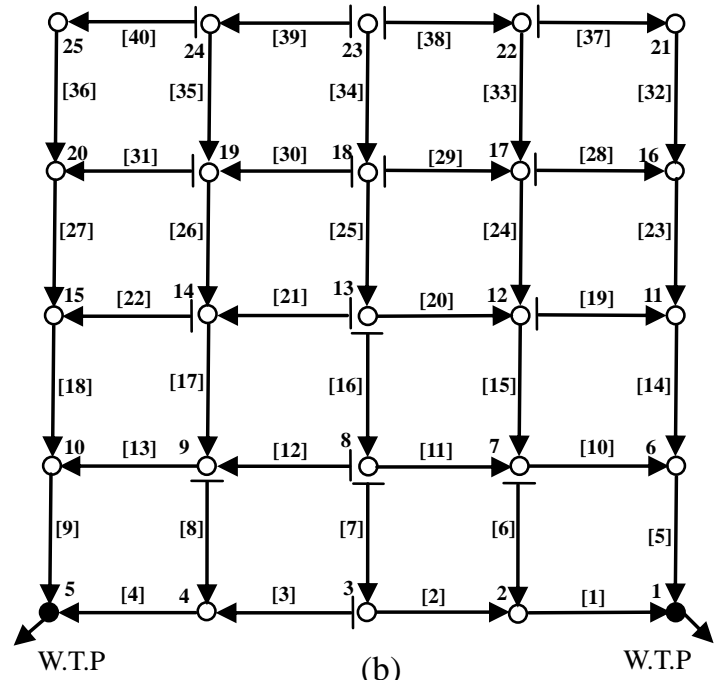

(b)

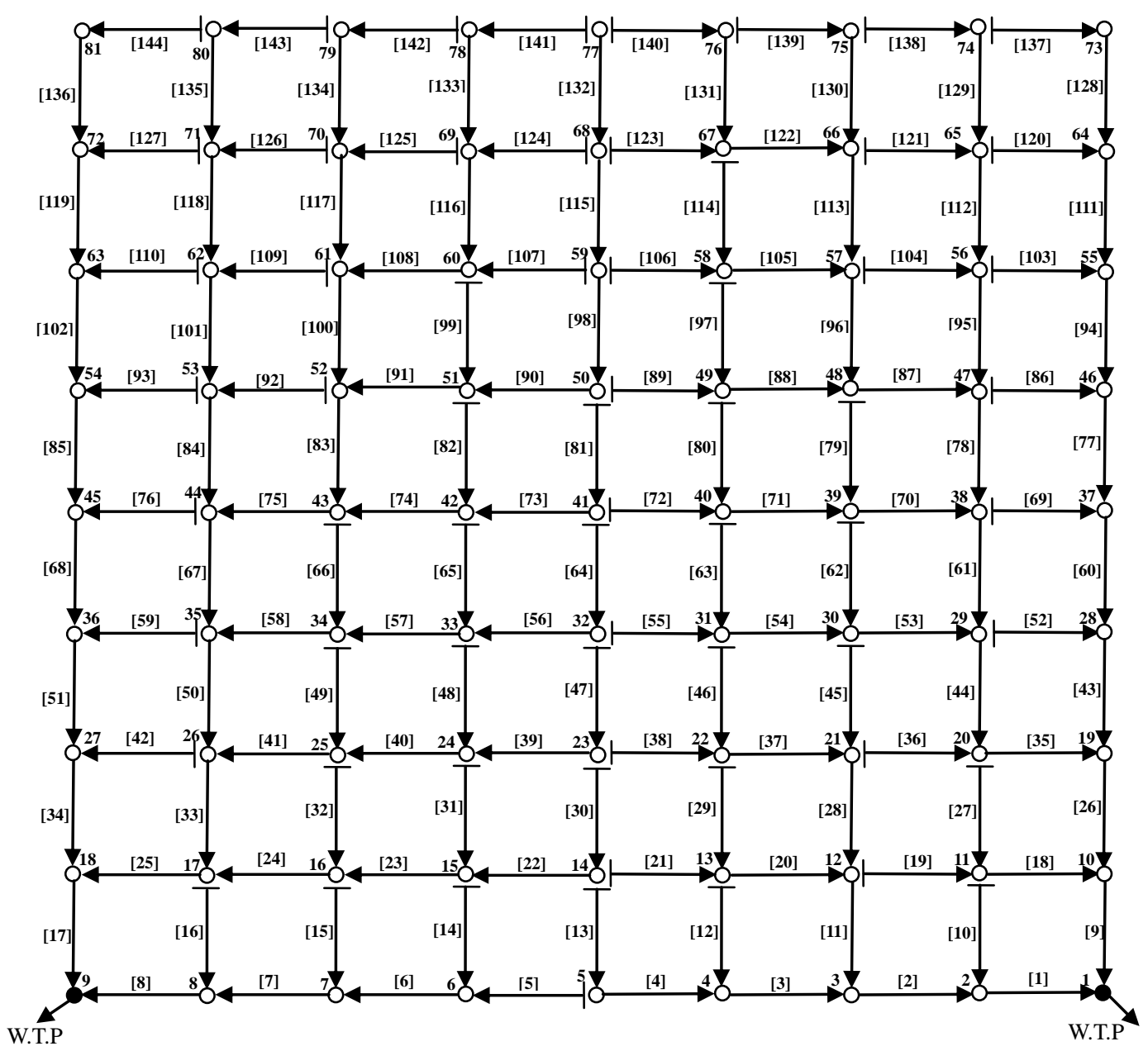

(c)

Figure 2. Network layouts for three examples: a) small scale sewer network, b) medium scale sewer network, c) large scale sewer network.

The optimal layouts obtained by Moeini and Afshar (2012 b), shown in Figure 2, is used here to assess the efficiency and effectiveness of the CA and HCA method. The small scale sewer network consists of 9 nodes and 12 pipes, the medium scale sewer network has 25 nodes and 40 pipes and the large scale sewer network includes 81 nodes and 144 pipes. All the networks have two treatment plants with fixed elevation located at the bottom corner of the sewer network. 
The ground elevation of a reference point located at the middle of the upper edge of the area denoted by node 8,23 and 77 in small, medium and large scale networks, respectively, is considered 1000 metres. The area is considered to have a constant slope of $2 \%$ from the reference point to the left and right and toward the bottom edge. The lengths of pipes in three networks are considered as 100 meters and the set of commercially available pipe diameters for all the pipes is included in the range of $100 \mathrm{~mm}$ to $1500 \mathrm{~mm}$ with an interval of $50 \mathrm{~mm}$ from $100 \mathrm{~mm}$ to $1000 \mathrm{~mm}$ and an interval of $100 \mathrm{~mm}$ from $1000 \mathrm{~mm}$ to $1500 \mathrm{~mm}$. The Manning coefficient is considered as 0.015 and the problem is constrained to a maximum and minimum velocity of $6 \mathrm{~m} / \mathrm{s}$ and $0.75 \mathrm{~m} / \mathrm{s}$, maximum and minimum cover depth of $10 \mathrm{~m}$ and $2.5 \mathrm{~m}$, and maximum and minimum relative flow depth of 0.83 and 0.1 , respectively.

The terms of pipe and manhole construction costs are defined as (Moeini and Afshar, $2012 \mathrm{a}, \mathrm{b}$ ):

$$
\begin{gathered}
K_{p}=10.93 e^{3.43 D}+0.012 X^{1.53}+0.437 X^{1.47} D \\
K_{h}=41.46 h_{m}
\end{gathered}
$$

Where, $D$ is the pipe diameter $(\mathrm{m}), X$ is the buried depth $(\mathrm{m})$, and $h_{m}$ is the depth of manhole (m).

These test problems are here solved using CA method of Afshar et al. (2011) and HCA methods and the results are presented and compared with other existing methods.

\begin{tabular}{|c|c|c|c|}
\hline Test Example & Model & Cost & Time (milli second) \\
\hline \multirow{5}{*}{ small scale network } & $\begin{array}{c}\text { ACOA-TGA } \\
\text { (Moeini and Afshar, } 2012 \mathrm{a} \text { ) }\end{array}$ & 23467 & - \\
\hline & $\begin{array}{c}\text { CACOA-TGA } \\
\text { (Moeini and Afshar, } 2012 \text { b) }\end{array}$ & 23467 & - \\
\hline & $\mathrm{CA}$ & 23811 & 1.6 \\
\hline & HCA-Discrete & 23460 & 4.7 \\
\hline & HCA-Continuous & 23513 & 3.1 \\
\hline \multirow{5}{*}{ medium scale network } & $\begin{array}{c}\text { ACOA-TGA } \\
\text { (Moeini and Afshar, } 2012 \text { a) }\end{array}$ & 86204 & - \\
\hline & $\begin{array}{c}\text { CACOA-TGA } \\
\text { (Moeini and Afshar, 2012 b) }\end{array}$ & 85990 & - \\
\hline & $\mathrm{CA}$ & 88096 & 14.1 \\
\hline & HCA-Discrete & 85873 & 46.9 \\
\hline & HCA- Continuous & 86678 & 15.6 \\
\hline \multirow{5}{*}{ large scale network } & $\begin{array}{c}\text { ACOA-TGA } \\
\text { (Moeini and Afshar, } 2012 \text { a) }\end{array}$ & 365600 & - \\
\hline & $\begin{array}{c}\text { CACOA-TGA } \\
\text { (Moeini and Afshar, } 2012 \text { b) }\end{array}$ & 363922 & - \\
\hline & $\mathrm{CA}$ & 370486 & 103.1 \\
\hline & HCA-Discrete & 361685 & 200.0 \\
\hline & HCA- Continuous & 367436 & 54.6 \\
\hline
\end{tabular}

Table 1. Optimal network cost obtained by different methods

Table 1 compares the optimal network costs and the CPU time required by the CA methods introduced here and those of ACOA-TGA (Moeini and Afshar, 2012 a) and CACOA-TGA (Moeini and Afshar, 2012 b) methods using a 2 MHz Pentium 4. It can be seen that both HCA methods produce better solutions than CA method in three sewer networks. Moreover, the HCA methods result in comparable solutions to the ACOA in small scale network, while with increasing the scale of the problem, the HCA methods produce superior solutions than ACOA. Furthermore, the HCA-discrete method results in better solution than the existing methods while requiring less than one second CPU time to achieve the solutions. It should be noted that ACOA needs much more time than CA methods, because of its mechanism and it is one the population based methods. Since the HCA method requires an initial guess for the decision variables of the problem, pipe diameters and the nodal cover depths, to start off the solution procedure, a sensitivity analysis is carried out here to assess the sensitivity of the final solution to the initial guess. 
Table 2. Maximum, Minimum and Average solution costs over 10 runs

\begin{tabular}{|c|c|c|c|c|c|}
\hline \multirow[b]{2}{*}{ Test Example } & \multicolumn{5}{|c|}{ Cost } \\
\hline & Model & Minimum & Maximum & Average & $\begin{array}{c}\text { Scaled Standard } \\
\text { Deviation }\end{array}$ \\
\hline \multirow{5}{*}{ small scale network } & $\begin{array}{c}\text { ACOA-TGA } \\
\text { (Moeini and Afshar, } 2012 \mathrm{a} \text { ) }\end{array}$ & 23467 & 23467 & 23467 & 0.0000 \\
\hline & $\begin{array}{c}\text { CACOA-TGA } \\
\text { (Moeini and Afshar, } 2012 \text { b) }\end{array}$ & 23467 & 23467 & 23467 & 0.0000 \\
\hline & $\mathrm{CA}$ & 23811 & 23811 & 23811 & 0.0000 \\
\hline & HCA-Discrete & 23460 & 23747 & 23546 & 0.0059 \\
\hline & HCA-Continuous & 23513 & 34064 & 25798 & 0.1683 \\
\hline \multirow{5}{*}{ medium scale network } & $\begin{array}{c}\text { ACOA-TGA } \\
\text { (Moeini and Afshar, } 2012 \text { a) }\end{array}$ & 86204 & 87127 & 86642 & 0.0037 \\
\hline & $\begin{array}{c}\text { CACOA-TGA } \\
\text { (Moeini and Afshar, } 2012 \text { b) }\end{array}$ & 85990 & 86591 & 86187 & 0.0020 \\
\hline & CA & 88096 & 88096 & 88096 & 0.0000 \\
\hline & HCA-Discrete & 85873 & 86953 & 86410 & 0.0052 \\
\hline & HCA- Continuous & 86678 & 87786 & 87397 & 0.0038 \\
\hline \multirow{5}{*}{ large scale network } & $\begin{array}{c}\text { ACOA-TGA } \\
\text { (Moeini and Afshar, } 2012 \text { a) }\end{array}$ & 365600 & 381484 & 372605 & 0.0127 \\
\hline & $\begin{array}{c}\text { CACOA-TGA } \\
\text { (Moeini and Afshar, } 2012 \text { b) }\end{array}$ & 363922 & 367174 & 365606 & 0.0030 \\
\hline & $\mathrm{CA}$ & 370486 & 370489 & 370488 & 0.0000 \\
\hline & HCA-Discrete & 361685 & 367131 & 363894 & 0.0040 \\
\hline & HCA-Continuous & 367436 & 371350 & 369661 & 0.0031 \\
\hline
\end{tabular}

Table 2 represents the maximum, minimum and average solution costs over 10 runs using different initial designs along with the scaled standard deviation of the solutions defined as the ratio of the standard deviation to the average solution. This table emphasizes on the insensitivity of the CA methods to the initial population.

Table 3. Results obtained from HCA method (discrete) for the small scale sewer network

\begin{tabular}{cccccc}
\hline \multicolumn{5}{c}{ Cover Depth $(\mathrm{m})$} \\
\hline Pipe & $\mathrm{D}(\mathrm{mm})$ & Upstream & Downstream & $\mathrm{V}(\mathrm{m} / \mathrm{s})$ & $\mathrm{y} / \mathrm{d}$ \\
\hline 1 & 100 & 2.50 & 4.50 & 1.39 & 0.57 \\
\hline 2 & 150 & 2.50 & 4.50 & 1.83 & 0.58 \\
\hline 3 & 200 & 3.40 & 4.50 & 2.04 & 0.67 \\
\hline 4 & 150 & 2.50 & 2.50 & 1.27 & 0.56 \\
\hline 5 & 200 & 2.50 & 4.50 & 2.08 & 0.50 \\
\hline 6 & 150 & 2.50 & 3.40 & 1.67 & 0.82 \\
\hline 7 & 150 & 2.50 & 2.50 & 1.27 & 0.56 \\
\hline 8 & 150 & 2.50 & 3.40 & 1.47 & 0.50 \\
\hline 9 & 150 & 2.50 & 2.50 & 1.27 & 0.56 \\
\hline 10 & 150 & 2.50 & 2.50 & 1.27 & 0.56 \\
\hline 11 & 100 & 2.50 & 2.50 & 1.05 & 0.73 \\
\hline 12 & 100 & 2.50 & 2.50 & 1.05 & 0.73 \\
\hline
\end{tabular}


Table 4. Results obtained from HCA method (discrete) for the medium scale sewer network

\begin{tabular}{|c|c|c|c|c|c|c|c|c|c|c|c|}
\hline \multicolumn{6}{|c|}{ Cover Depth (m) } & \multicolumn{6}{|c|}{ Cover Depth (m) } \\
\hline Pipe & $\mathrm{D}(\mathrm{mm})$ & Upstream & Downstream & $\mathrm{V}(\mathrm{m} / \mathrm{s})$ & $\mathrm{y} / \mathrm{d}$ & Pipe & $\mathrm{D}(\mathrm{mm})$ & Upstream & Downstream & $\mathrm{V}(\mathrm{m} / \mathrm{s})$ & $\mathrm{y} / \mathrm{d}$ \\
\hline 1 & 200 & 2.50 & 4.50 & 2.18 & 0.55 & 21 & 150 & 2.50 & 2.50 & 1.27 & 0.56 \\
\hline 2 & 150 & 2.50 & 2.50 & 1.38 & 0.74 & 22 & 150 & 2.50 & 2.50 & 1.27 & 0.56 \\
\hline 3 & 100 & 2.50 & 2.50 & 1.05 & 0.73 & 23 & 200 & 2.50 & 2.50 & 1.60 & 0.62 \\
\hline 4 & 150 & 2.50 & 4.50 & 1.94 & 0.71 & 24 & 200 & 2.50 & 2.80 & 1.79 & 0.75 \\
\hline 5 & 350 & 3.45 & 4.50 & 2.92 & 0.65 & 25 & 150 & 2.50 & 3.40 & 1.67 & 0.82 \\
\hline 6 & 150 & 2.50 & 2.50 & 1.27 & 0.56 & 26 & 200 & 2.50 & 2.50 & 1.68 & 0.80 \\
\hline 7 & 150 & 2.50 & 2.50 & 1.27 & 0.56 & 27 & 200 & 2.50 & 2.50 & 1.60 & 0.62 \\
\hline 8 & 150 & 2.50 & 2.50 & 1.27 & 0.56 & 28 & 150 & 2.50 & 2.50 & 1.27 & 0.56 \\
\hline 9 & 300 & 3.17 & 4.50 & 2.82 & 0.73 & 29 & 150 & 2.50 & 2.50 & 1.27 & 0.56 \\
\hline 10 & 300 & 3.47 & 3.45 & 2.19 & 0.82 & 30 & 150 & 2.50 & 2.50 & 1.27 & 0.56 \\
\hline 11 & 150 & 2.50 & 3.47 & 1.69 & 0.81 & 31 & 150 & 2.50 & 2.50 & 1.27 & 0.56 \\
\hline 12 & 150 & 2.50 & 2.50 & 1.27 & 0.56 & 32 & 150 & 2.50 & 2.50 & 1.27 & 0.56 \\
\hline 13 & 250 & 2.50 & 3.17 & 2.26 & 0.82 & 33 & 150 & 2.50 & 2.50 & 1.38 & 0.74 \\
\hline 14 & 200 & 2.50 & 3.45 & 2.03 & 0.76 & 34 & 150 & 2.50 & 2.50 & 1.27 & 0.56 \\
\hline 15 & 250 & 2.80 & 3.47 & 2.26 & 0.82 & 35 & 150 & 2.50 & 2.50 & 1.38 & 0.74 \\
\hline 16 & 150 & 2.50 & 2.50 & 1.27 & 0.56 & 36 & 150 & 2.50 & 2.50 & 1.27 & 0.56 \\
\hline 17 & 250 & 2.50 & 2.50 & 1.92 & 0.71 & 37 & 100 & 2.50 & 2.50 & 1.05 & 0.73 \\
\hline 18 & 200 & 2.50 & 3.17 & 1.94 & 0.79 & 38 & 100 & 2.50 & 2.50 & 1.05 & 0.73 \\
\hline 19 & 150 & 2.50 & 2.50 & 1.27 & 0.56 & 39 & 100 & 2.50 & 2.50 & 1.05 & 0.73 \\
\hline 20 & 200 & 3.40 & 2.80 & 1.41 & 0.82 & 40 & 100 & 2.50 & 2.50 & 1.05 & 0.73 \\
\hline
\end{tabular}

Table 5. Results obtained from HCA method (discrete) for the large scale sewer network

\begin{tabular}{|c|c|c|c|c|c|c|c|c|c|c|c|}
\hline \multicolumn{6}{|c|}{ Cover Depth (m) } & \multicolumn{6}{|c|}{ Cover Depth (m) } \\
\hline Pipe & $\mathrm{D}(\mathrm{mm})$ & Upstream & Downstream & $\mathrm{V}(\mathrm{m} / \mathrm{s})$ & $\mathrm{y} / \mathrm{d}$ & Pipe & $\mathrm{D}(\mathrm{mm})$ & Upstream & Downstream & $\mathrm{V}(\mathrm{m} / \mathrm{s})$ & $\mathrm{y} / \mathrm{d}$ \\
\hline 1 & 350 & 4.14 & 4.50 & 2.59 & 0.68 & 73 & 200 & 2.50 & 2.50 & 1.52 & 0.53 \\
\hline 2 & 300 & 3.34 & 4.14 & 2.61 & 0.82 & 74 & 200 & 2.50 & 3.00 & 1.88 & 0.82 \\
\hline 3 & 200 & 2.50 & 3.34 & 1.90 & 0.62 & 75 & 400 & 3.00 & 3.17 & 2.77 & 0.76 \\
\hline 4 & 150 & 2.50 & 2.50 & 1.38 & 0.74 & 76 & 150 & 2.50 & 2.50 & 1.27 & 0.56 \\
\hline 5 & 100 & 2.50 & 2.50 & 1.05 & 0.73 & 77 & 250 & 3.00 & 2.50 & 1.69 & 0.80 \\
\hline 6 & 200 & 2.50 & 2.50 & 1.52 & 0.53 & 78 & 400 & 4.02 & 4.37 & 2.84 & 0.70 \\
\hline 7 & 200 & 2.50 & 2.50 & 1.68 & 0.80 & 79 & 150 & 2.50 & 2.50 & 1.27 & 0.56 \\
\hline 8 & 200 & 2.50 & 4.50 & 2.38 & 0.81 & 80 & 150 & 2.50 & 2.50 & 1.27 & 0.56 \\
\hline 9 & 500 & 3.93 & 4.50 & 3.52 & 0.82 & 81 & 150 & 2.50 & 2.50 & 1.27 & 0.56 \\
\hline 10 & 150 & 2.50 & 4.14 & 1.60 & 0.47 & 82 & 150 & 2.50 & 2.50 & 1.27 & 0.56 \\
\hline 11 & 300 & 3.88 & 3.34 & 1.88 & 0.82 & 83 & 350 & 2.71 & 3.00 & 2.62 & 0.82 \\
\hline 12 & 150 & 2.50 & 2.50 & 1.27 & 0.56 & 84 & 250 & 2.50 & 3.17 & 2.26 & 0.82 \\
\hline 13 & 150 & 2.50 & 2.50 & 1.27 & 0.56 & 85 & 250 & 3.00 & 2.50 & 1.69 & 0.80 \\
\hline 14 & 150 & 2.50 & 2.50 & 1.27 & 0.56 & 86 & 150 & 2.50 & 3.00 & 1.39 & 0.52 \\
\hline 15 & 150 & 2.50 & 2.50 & 1.27 & 0.56 & 87 & 300 & 2.50 & 4.02 & 2.93 & 0.82 \\
\hline 16 & 150 & 2.50 & 2.50 & 1.27 & 0.56 & 88 & 200 & 2.50 & 2.50 & 1.65 & 0.70 \\
\hline 17 & 550 & 3.78 & 4.50 & 3.86 & 0.82 & 89 & 150 & 2.50 & 2.50 & 1.27 & 0.56 \\
\hline 18 & 200 & 2.50 & 3.93 & 2.05 & 0.58 & 90 & 200 & 2.50 & 3.00 & 1.88 & 0.82 \\
\hline 19 & 150 & 2.50 & 2.50 & 1.27 & 0.56 & 91 & 250 & 3.00 & 2.71 & 1.81 & 0.82 \\
\hline 20 & 200 & 2.50 & 3.88 & 2.04 & 0.58 & 92 & 150 & 2.50 & 2.50 & 1.27 & 0.56 \\
\hline 21 & 150 & 2.50 & 2.50 & 1.27 & 0.56 & 93 & 150 & 2.50 & 3.00 & 1.39 & 0.52 \\
\hline 22 & 150 & 2.50 & 3.40 & 1.67 & 0.82 & 94 & 200 & 2.50 & 3.00 & 1.88 & 0.82 \\
\hline 23 & 200 & 3.40 & 3.89 & 1.88 & 0.82 & 95 & 250 & 2.50 & 4.02 & 2.41 & 0.58 \\
\hline 24 & 250 & 3.89 & 3.60 & 1.81 & 0.82 & 96 & 300 & 2.50 & 2.50 & 2.20 & 0.78 \\
\hline 25 & 550 & 3.60 & 3.78 & 3.43 & 0.76 & 97 & 150 & 2.50 & 2.50 & 1.27 & 0.56 \\
\hline 26 & 500 & 3.71 & 3.93 & 3.26 & 0.82 & 98 & 200 & 2.50 & 2.50 & 1.65 & 0.70 \\
\hline 27 & 150 & 2.50 & 2.50 & 1.27 & 0.56 & 99 & 150 & 2.50 & 3.00 & 1.39 & 0.52 \\
\hline 28 & 200 & 2.50 & 3.88 & 1.77 & 0.78 & 100 & 300 & 2.5 & 2.71 & 2.30 & 0.74 \\
\hline 29 & 150 & 2.50 & 2.50 & 1.27 & 0.56 & 101 & 250 & 2.50 & 2.50 & 1.92 & 0.71 \\
\hline
\end{tabular}




\begin{tabular}{|c|c|c|c|c|c|c|c|c|c|c|c|}
\hline 30 & 150 & 2.50 & 2.50 & 1.27 & 0.56 & 102 & 200 & 2.50 & 3.00 & 1.88 & 0.82 \\
\hline 31 & 150 & 2.50 & 3.40 & 1.47 & 0.50 & 103 & 150 & 2.50 & 2.50 & 1.27 & 0.56 \\
\hline 32 & 150 & 2.50 & 3.89 & 1.56 & 0.48 & 104 & 150 & 2.50 & 2.50 & 1.27 & 0.56 \\
\hline 33 & 550 & 4.00 & 3.60 & 2.95 & 0.77 & 105 & 200 & 2.50 & 2.50 & 1.65 & 0.70 \\
\hline 34 & 300 & 2.50 & 3.78 & 2.71 & 0.64 & 106 & 150 & 2.50 & 2.50 & 1.27 & 0.56 \\
\hline 35 & 500 & 4.32 & 3.71 & 2.59 & 0.82 & 107 & 150 & 2.50 & 2.50 & 1.27 & 0.56 \\
\hline 36 & 150 & 2.50 & 4.32 & 1.63 & 0.46 & 108 & 250 & 2.50 & 2.50 & 1.92 & 0.71 \\
\hline 37 & 200 & 2.50 & 2.50 & 1.65 & 0.70 & 109 & 150 & 2.50 & 2.50 & 1.27 & 0.56 \\
\hline 38 & 150 & 2.50 & 2.50 & 1.27 & 0.56 & 110 & 150 & 2.50 & 2.50 & 1.27 & 0.56 \\
\hline 39 & 200 & 2.50 & 2.50 & 1.52 & 0.53 & 111 & 200 & 2.50 & 2.50 & 1.60 & 0.62 \\
\hline 40 & 200 & 2.50 & 3.00 & 1.88 & 0.82 & 112 & 200 & 2.50 & 2.50 & 1.68 & 0.80 \\
\hline 41 & 250 & 3.00 & 4.00 & 2.31 & 0.65 & 113 & 250 & 2.50 & 2.50 & 1.94 & 0.76 \\
\hline 42 & 150 & 2.50 & 2.50 & 1.27 & 0.56 & 114 & 150 & 2.50 & 2.50 & 1.27 & 0.56 \\
\hline 43 & 300 & 2.50 & 3.71 & 2.60 & 0.58 & 115 & 200 & 2.50 & 2.50 & 1.52 & 0.53 \\
\hline 44 & 500 & 5.08 & 4.32 & 2.44 & 0.82 & 116 & 200 & 2.50 & 2.50 & 1.68 & 0.80 \\
\hline 45 & 150 & 2.50 & 2.50 & 1.27 & 0.56 & 117 & 200 & 2.50 & 2.50 & 1.68 & 0.80 \\
\hline 46 & 150 & 2.50 & 2.50 & 1.27 & 0.56 & 118 & 200 & 2.50 & 2.50 & 1.68 & 0.80 \\
\hline 47 & 150 & 2.50 & 2.50 & 1.27 & 0.56 & 119 & 200 & 2.50 & 2.50 & 1.60 & 0.62 \\
\hline 48 & 150 & 2.50 & 2.50 & 1.27 & 0.56 & 120 & 150 & 2.50 & 2.50 & 1.27 & 0.56 \\
\hline 49 & 150 & 2.50 & 3.00 & 1.39 & 0.52 & 121 & 150 & 2.50 & 2.50 & 1.27 & 0.56 \\
\hline 50 & 450 & 3.09 & 4.00 & 3.48 & 0.82 & 122 & 200 & 2.50 & 2.50 & 1.68 & 0.80 \\
\hline 51 & 300 & 2.83 & 2.50 & 2.00 & 0.73 & 123 & 150 & 2.50 & 2.50 & 1.27 & 0.56 \\
\hline 52 & 150 & 2.50 & 2.50 & 1.27 & 0.56 & 124 & 150 & 2.50 & 2.50 & 1.27 & 0.56 \\
\hline 53 & 200 & 2.50 & 5.08 & 2.54 & 0.76 & 125 & 150 & 2.50 & 2.50 & 1.27 & 0.56 \\
\hline 54 & 200 & 2.50 & 2.50 & 1.65 & 0.70 & 126 & 150 & 2.50 & 2.50 & 1.27 & 0.56 \\
\hline 55 & 150 & 2.50 & 2.50 & 1.27 & 0.56 & 127 & 150 & 2.50 & 2.50 & 1.27 & 0.56 \\
\hline 56 & 150 & 2.50 & 3.40 & 1.67 & 0.82 & 128 & 150 & 2.50 & 2.50 & 1.27 & 0.56 \\
\hline 57 & 250 & 3.40 & 2.50 & 1.42 & 0.70 & 129 & 150 & 2.50 & 2.50 & 1.38 & 0.74 \\
\hline 58 & 250 & 2.50 & 3.09 & 2.17 & 0.68 & 130 & 150 & 2.50 & 2.50 & 1.38 & 0.74 \\
\hline 59 & 150 & 2.50 & 2.83 & 1.35 & 0.53 & 131 & 150 & 2.50 & 2.50 & 1.38 & 0.74 \\
\hline 60 & 300 & 2.50 & 2.50 & 2.07 & 0.60 & 132 & 150 & 2.50 & 2.50 & 1.27 & 0.56 \\
\hline 61 & 400 & 4.37 & 5.08 & 3.11 & 0.82 & 133 & 150 & 2.50 & 2.50 & 1.38 & 0.74 \\
\hline 62 & 150 & 2.50 & 2.50 & 1.27 & 0.56 & 134 & 150 & 2.50 & 2.50 & 1.38 & 0.74 \\
\hline 63 & 150 & 2.50 & 2.50 & 1.27 & 0.56 & 135 & 150 & 2.50 & 2.50 & 1.38 & 0.74 \\
\hline 64 & 150 & 2.50 & 2.50 & 1.27 & 0.56 & 136 & 150 & 2.50 & 2.50 & 1.27 & 0.56 \\
\hline 65 & 150 & 2.50 & 3.40 & 1.47 & 0.50 & 137 & 100 & 2.50 & 2.50 & 1.05 & 0.73 \\
\hline 66 & 150 & 2.50 & 2.50 & 1.27 & 0.56 & 138 & 100 & 2.50 & 2.50 & 1.05 & 0.73 \\
\hline 67 & 450 & 3.17 & 3.09 & 2.83 & 0.82 & 139 & 100 & 2.50 & 2.50 & 1.05 & 0.73 \\
\hline 68 & 250 & 2.50 & 2.83 & 2.11 & 0.82 & 140 & 100 & 2.50 & 2.50 & 1.05 & 0.73 \\
\hline 69 & 150 & 2.50 & 2.50 & 1.27 & 0.56 & 141 & 100 & 2.50 & 2.50 & 1.05 & 0.73 \\
\hline 70 & 200 & 2.50 & 4.37 & 2.34 & 0.82 & 142 & 100 & 2.50 & 2.50 & 1.05 & 0.73 \\
\hline 71 & 200 & 2.50 & 2.50 & 1.65 & 0.70 & 143 & 100 & 2.50 & 2.50 & 1.05 & 0.73 \\
\hline 72 & 150 & 2.50 & 2.50 & 1.27 & 0.56 & 144 & 100 & 2.50 & 2.50 & 1.05 & 0.73 \\
\hline
\end{tabular}

Details of the optimal solution obtained by the HCA-discrete method for three sewer networks are also shown in Table 3,4 , and 5 , respectively.

\section{Concluding Remarks}

In this paper, Hybrid Cellular Automata approach was used for the optimal solution of sewer network design problems. The problem was decomposed to two stages with considering the nodal cover depths and pipe diameters as decision variables. In the first stage, nodal cover depths were calculated assuming fixed values for the pipe diameters, while in the second stage, the pipe diameters were determined in two approaches of continuous and discrete with nodal cover depths of the first stage. Two stages were solved iteratively until the convergence was achieved. The HCA methods were used to solve three benchmark examples in the literature and the comparison of results with two versions of Ant Colony Optimization Algorithm indicated the ability and efficiency of the HCA methods to produce better results comparable to those of heuristic search methods with much higher efficiency. 


\section{References}

Adamatzky, A. I. (1996). Computation of shortest path in cellular automata. Mathematical and Computer Modelling, 23(4), 105-113.

Afshar, M. H. (2007). Partially Constrained Ant Colony Optimization Algorithm for the solution of constrained optimization problems: application to storm water network design. Adv Water Resour, 30, 954-965.

Afshar, M. H., \& Shahidi, M. (2009). Optimal solution of large scale reservoir operation problems: Cellular Automata versus heuristic-search methods. Engineering Optimization, 41(3), 275-293.

Afshar, M. H. (2010). A parameter free Continuous Ant Colony Optimization Algorithm for the optimal design of storm sewer networks: Constrained and unconstrained approach. Advances in Engineering Software, 41(2), 188-195.

Afshar, M. H., Shahidi, M., Rohani, M., \& Sargolzaei, M. (2011). Application of cellular automata to sewer network optimization problems. Scientia Iranica A, 18(3), 304-312.

Afshar, M. H., \& Rohani, M. (2012). Optimal design of sewer networks using cellular automata-based hybrid methods: Discrete and continuous approaches. Engineering Optimization, 44(1), 1-22.

Burks, E. (1972). Essays on Cellular Automat, University of Illinois Press, Champaign, IL.

Codd, E F. (1968). Cellular Automata, Academic Press, New York.

Dajani, J. S., \& Gemmel, R. S. (1971). Economics of wastewater collection networks, Civil Eng. Res. Rep. 43, Water Resour. Center, Univ. of Ill. At Urbana-Champaign, Urbana.

Deininger, R. A. (1970). Systems analysis for water supply and pollution control. Natural Resource Systems Models in decision Making, edited by G.H. Toebes, Water Resources Center, Purdue Univ. Lafayette, Ind., 45-65.

El Dessouki, W. M., Fathi, Y., \& Rouphail, N. (2001). Meta-optimization using cellular automata with application to the combined trip distribution and assignment system optimal problem. Computer-Aided Civil and Infrastructure Engineering, 16(6), 384-396.

Froise, S., \& Burges, S. J. (1978). Least-cost design of urban drainage networks. Water Resources Planning and Management Division, ASCE, 104(1), 75-92.

Guo, Y., Walters, G. A., Khu, S. T., \& Keedwell, E. (2007a). A novel cellular automata based approach to storm sewer design. Engineering Optimization, 39(3), 345-364.

Guo, Y., Keedwell, E. C., Walters, G. A., \& Khu, S. T. (2007b). Hybridizing Cellular Automata Principles and NSGAII for Multi-objective Design of Urban Water Networks. Evolutionary Multi-Criterion Optimization, 546-559.

Gupta, J. M., Agarwal, S. K., \& Khanna, P. (1976). Optimal design of wastewater collection systems. The Environmental Engineering Division, 102(EE5), 1029-1041.

Heaney, J. P., Wright, L. T., Sample, D., Field, R., \& Fan, C. Y. (1999). Innovative methods for the optimization of gravity storm sewer design. Proceedings the 8th international conference on urban storm drainage, Sydney, Australia, 1896-1903.

Keedwell, E., \& Khu, S. T. (2005). Using cellular automata to seed genetic algorithms for water distribution network design problems. Engineering Applications of Artificial Intelligence, 18(4), 461-472.

Kita, E., \& Toyoda, T. (2000). Structural design using cellular automata. Structural and Multidisciplinary Optimization, 19, 64-73.

Kulkarni, V. S., \& Khanna, P. (1985). Pumped wastewater collection systems optimization. Environ Eng, ASCE, 111(5), 589-601.

Lemieux, P. F., Zech, Y., \& Delarue, R. (1976). Design of a stormwater sewer by nonlinear programming. Canadian Journal of Civil Engineering, 3(1), 83-89.

Liang, L.Y., Thompson, R. G., \& Young, D.M. (2004). Optimizing the design of sewer networks using genetic algorithms and tabu search. Engineering, Construction and Architectural Management, 11(2), 101-112.

Mays, L. W., \& Wenzel, H. G. (1976). Optimal design of multi-level branching sewer systems. Water Resources Research, 12(5), 913-917.

Merrit, L. B., \& Bogan, R. H. (1973). Computerbase Optimal Design of Sewer System. The Environmental Engineering Division, ASCE, 99(EE1), 35-53.

Missoum, S., Gürdal, Z., \& Setoodeh, S. (2005). Study of a new local update scheme for cellular automata in structural design. Struct. Multidisciplinary Optimization, 29, 103-112. 
Moeini, R., \& Afshar, M. H. (2012a). Layout and size optimization of sanitary sewer network using intelligent ants. Advances in Engineering Software, 51, 49-61.

Moeini, R., \& Afshar, M. H. (2012b). Constrained Ant Colony Optimisation Algorithm for the layout and size optimisation of sanitary sewer networks. Urban Water Journal, 1-20.

Packard, N. H. (1986). Lattice Models for Solidification and Aggregation. In First International Symosium for Science on Form.

Pan, T. C., \& Kao, J. J. (2009). GA-QP model to optimize sewer system design. Environmental Engineering, 135(1), $17-24$.

Price, R. K. (1978). Design of storm water sewers for minimum construction cost. In Proc. $1^{\text {st }}$ International Conference on Urban Strom Drainage, Southampton, United Kingdom, 636-647.

Robinson, D. K., \& Labadie, J. W. (1981). Optimal design of urban storm water drainage system. International symposium on urban hydrology, hydraulics, and sediment control, University of Kentucky Lexington, 145-56.

Sentos, R. M. Z. D., \& Coutinho S. (2001). Dynamics of HIV Approach: A Cellular Automata Approach. Phys. Rev. Lett., 87(16), 102-104.

Setoodeh, S., Gürdalb, Z., \& Watson, L. T. (2006). Design of variable-stiffness composite layers using cellular automata. Computer Methods in Applied Mechanics and Engineering, 195(9-12), 836-851.

Shuai, D., \& Zhao, H. (2004). A new generalized cellular automata approach to optimization of fast packet switching. Computer Networks, 45, 399-419.

Swamee, P. K. (2001). Design of sewer line. Environmental Engineering, 127(9), 776-781.

Thatcher, J. (1964). Universality in the von Neumann cellular model. Technical Report 03105-30-T, University of Michigan.

Ulam, S. (1960). A collection of mathematical problems. NewYork, Interscience publishers.

Von Neumann, J. (1966). Theory of self-reproducing automata. Champaign, IL, University of Illinois Press.

Walters, G. A., \& Templeman, A. B. (1979). Non-optimal dynamic programming algorithms in the design of minimum cost drainage systems. Engineering Optimization, 4, 139-148.

Wang, L., Zhou, Y. (2009). Comparative Study on Bionic Optimization Algorithms for Sewer Optimal Design. 2009 Fifth International Conference on Natural Computation, 24-29.

Wolfram, S. (1988). High Speed Computing: Scientific Application and Algorithm Design. University of Illinois Press.

Yeh, S. F., Chu, C. W., Chang, Y. J., \& Lin, M. D. (2011). Applying tabu search and simulated annealing to the optimal design of sewer networks. Engineering Optimization, 43(2), 159-174.

Yen, B. C., Cheng, S. T., Jun, B. H., Voohees, M. L., \& Wenzel, H. G. (1984). Illinois least cost sewer system design model. User's guide, Department of Civil Engineering, University of Texas at Austin.

\section{$($ (c) $)$ EY}

This work is licensed under a Creative Commons Attribution 3.0 License. 